\title{
State of Health Monitoring of a Battery Module Using Multilayer Neural Network and Internal Resistance
}

\author{
Jong-Hyun Lee ${ }^{1}$, Hyun-Sil Kim², and In-Soo Lee ${ }^{1,3}$ \\ ${ }^{1}$ School of Electronic and Electrical Engineering Kyungpook National University, Korea. \\ ${ }^{2}$ Naval Combat Systems PMO Agency For Defence Development, Korea. \\ ${ }^{3}$ Corresponding author, School of Electronics Engineering Kyungpook National University, Korea. \\ ${ }^{3}$ ORCID: 0000-0001-9773-4710
}

\begin{abstract}
Lithium batteries are presently used in various applications, such as cell phones, electric vehicles, unmanned submarines, and energy storage systems, as main power sources. Therefore, for stable and safe use of this system, it is important to rapidly detect defects in the battery and accurately diagnose faults. Battery faults can be diagnosed by measuring their state of health $(\mathrm{SOH})$, which is affected by various operating conditions. In this work, a battery SOH monitoring system is implemented to detect faults using a multilayer neural network state classifier (MNNSC) and an internal resistance state classifier (IRSC). In this system, the MNNSC uses discharge voltage data from a lithium battery operating at high temperatures. Further, the IRSC uses the open circuit voltage, terminal voltage, and current to calculate the internal resistance. Experimental results show that the proposed battery SOH monitoring method was high accuracy.
\end{abstract}

Keywords: Lithium battery, State of health, Fault diagnosis system, Multilayer neural network, Internal resistance

\section{INTRODUCTION}

Lithium batteries are one of several types of devices used to store energy these days, which have attracted significant attention from users and researchers owing to their high energy and power density, long lifetime, and environmental friendliness [1]. Lithium batteries are used in applications such as cell phones, electric vehicles, unmanned submarines, and energy storage systems as the main sources of power [2, 3]. However, faults in the battery system can degrade device performance and even cause serious operational faults $[4,5]$. As the risk of battery system faults have increased in recent years, research on their fault diagnosis and safety management has, therefore, become particularly important $[6,7]$. There are several methods to diagnose faults in batteries; however, the status of a battery can generally be diagnosed by measuring its state of health ( $\mathrm{SOH})$. Existing methods typically determine the $\mathrm{SOH}$ of a battery based on the lifetime specified by the manufacturer or on the number of charge-discharge cycles, however, the lifetime of the lithium battery can be reduced, or faults may occur earlier, depending on the temperature and operating conditions.

Currently, the field of estimating or diagnosing battery aging using $\mathrm{SOH}$ is as follows: $\mathrm{EV}$ (Electric Vehicles), drones,
ESS(Energy Storage System) [8, 9]. In this field, since the capacity reduction of the battery affects the performance, it is important to know when to replace the battery early by using the SOH of the battery.

Today, various methods of $\mathrm{SOH}$ estimation research are in progress. The $\mathrm{SOH}$ estimation methods are mainly the open circuit voltage $(\mathrm{OCV})$ and Kalman filter methods. The Kalman filter method is used to predict the current state of the battery by predicting the internal resistance of the battery [10]. However, the Kalman filter method has the disadvantage of being difficult to apply, owing to the complexities of its parameters and algorithms. The open circuit voltage (OCV) method measures the output voltage of the battery under noload conditions and predicts the $\mathrm{SOH}$ according to the changes in the OCV [11]. However, it is difficult to apply the OCV method to real-time systems as measurements need to be obtained when the battery is in a state of internal chemical equilibrium. The SOH prediction using the RNN method feeds the previous parameters back and makes predictions using past information and the current data. However, learning from previous data is slow and the resulting low operation speed is a disadvantage [12-13].

Nowadays, neural networks have been applied in various tasks, such pattern recognition, identification, and classification. The neural networks can be characterized by the following three representative features. First, neural networks have the ability to learn complex nonlinear input-output relationships. Second, neural networks can use the sequential training procedures. Third, neural networks allow data adaptability. Neural networks have proven promising for machine intelligence by allowing the construction of algorithms that can learn from the input and output data and can perform data-driven decisions or predictions [14-15] Furthermore, neural networks can be used for efficient modelling of a variety of input-output relationships, and possess the advantage of shorter execution times compared to procedural models [16-17].

In this work, we propose an SOH monitoring method for a battery module using a multilayer neural network (MNN) and the internal resistance of the battery. The lithium battery characteristics change according to temperature. Therefore, when diagnosing the $\mathrm{SOH}$, it is necessary to consider the change in characteristics of the battery by temperature. [18-19] In this study, to diagnose its state the battery was operated at a high temperature $\left(40^{\circ} \mathrm{C}\right)$ for an extended period of time. Using experimental data, the battery faults were diagnosed by a 
multilayer neural network state classifier (MNNSC) and an internal resistance state classifier (IRSC).

\section{MONITORING SYSTEM BASED ON MNNSC AND IRSC}

\section{II.1 Lithium battery experimental setup}

The configuration of the battery experiment environment in this study is as follows [20]. Battery data are obtained by charging and discharging the battery in a chamber under a high temperature, i.e., $40^{\circ} \mathrm{C}$ in this experimental environment. The discharge of the battery proceeded to $1 \mathrm{C}$, where $\mathrm{C}$ is the current rate. The current rate is a measure of the rate at which a battery is discharged relative to its maximum capacity. A $1 \mathrm{C}$ rate implies that the discharge current will discharge the entire battery in $1 \mathrm{~h}$.

\section{2 Battery SOH Algorithm}

The configuration of the lithium battery $\mathrm{SOH}$ monitoring system proposed in this study is shown in Fig 1. The proposed system comprises a battery controller, MNNSC, IRSC, and coordinator. The battery controller comprises a voltage and current sensor, battery protection circuit, and micro controller unit (MCU). It measures the voltage and current data when charging or discharging the battery and sends the data to the PC. The MNNSC is a state classifier that uses a multilayer neural network. The MNNSC receives voltage data from the battery controller and diagnoses the battery status as one of the three states: normal, warning, and fault [20]. The IRSC diagnoses normal or abnormal state by comparing the measured resistance to a threshold. Finally, based on the inputs from the MNNSC and IRSC, the coordinator outputs the diagnosis result as normal, warning, or fault, respectively.

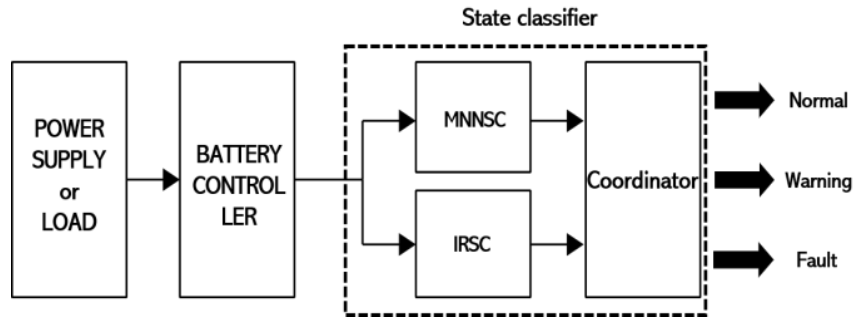

Fig. 1. Configuration of the battery SOH monitoring system.

The fault diagnosis process of the battery proceeds as shown in Fig. 2. First, the OCV is measured, then the load voltage and load current are obtained, and the estimated resistance of the equivalent circuit model of the battery is calculated using Equation (1)-(4). Second, the input discharge voltage data are classified as normal, warning, or fault by the MNNSC. Further, the input parameters, such as OCV, current and discharge voltage, are input to the IRSC, whose output is either a normal or abnormal state indication. Finally, the coordinator categorizes the battery state as normal, warning, or fault using the outputs of the MNNSC and IRSC, and diagnostic rules. The coordinator diagnostic rules are shown in Table 1.

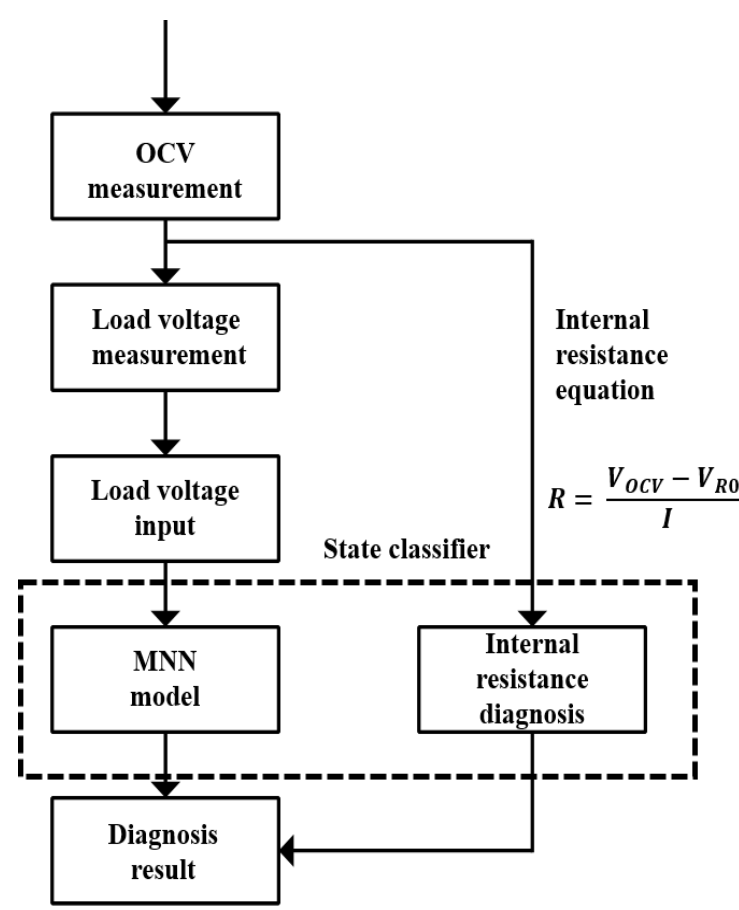

Fig. 2. Process of battery SOH diagnosis.

Table. 1. The diagnostic rules for determining coordinator results

\begin{tabular}{ccc}
\hline MNNSC result & IRSC result & Coordinator result \\
\hline Normal & Normal & Normal \\
Normal & Abnormal & Warning \\
Warning & Normal or Abnormal & Warning \\
Fault & Normal or Abnormal & Fault \\
\hline
\end{tabular}

\section{1) Internal Resistance State Classifier}

In this work, the internal parameters of the battery are estimated using its equivalent circuit model shown in Fig. 3 [21].

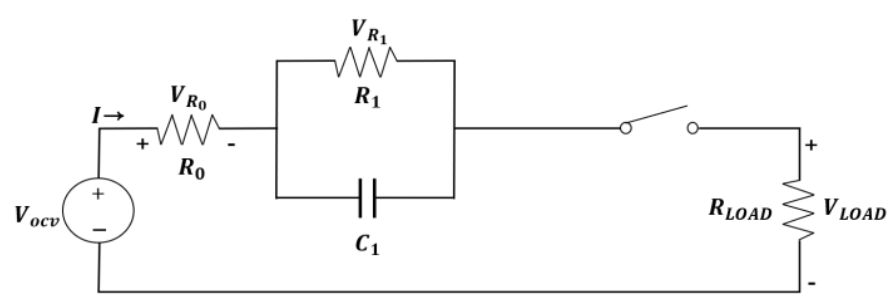

Fig. 3. Equivalent circuit model of battery.

In this model, $V_{O C V}$ is the OCV of the battery, $V_{L O A D}$ is the battery load voltage, and $\mathrm{V}_{R 0}$ and $\mathrm{V}_{R 1}$ are the voltages across $R_{0}$ and $R_{1}$, respectively, $V_{O C V}$ is obtained as follows:

$$
V_{O C V}=V_{L O A D}+\mathrm{V}_{R 0}+\mathrm{V}_{R 1}
$$


At the moment when the circuit is closed, $\mathrm{V}_{R 1}$ becomes 0 , because of the capacitor $\mathrm{C} 1$, and $V_{R 0}$ is calculated as follows:

$$
V_{R 0}=V_{O C V}-V_{L O A D}
$$

According to Ohm's Law, the $V_{R 0}$ is expressed as follows:

$$
\mathrm{I} \times R_{0}=V_{O C V}-\mathrm{V}_{L O A D}
$$

The battery state is estimated by measuring its internal resistance, $R_{0}$, which is calculated as follows:

$$
R_{0}=\frac{V_{O C V}-V_{L O A D}}{\mathrm{I}}
$$

As the battery ages, its internal resistance increases, therefore, when the SOH of the battery reduces to $0 \%$, the values of the internal resistances, $R_{0}$ and $R_{1}$, are higher than those when the $\mathrm{SOH}$ is $100 \%$. In other words, the internal resistance of the battery can be measured and used to diagnose its state. The internal resistance is defined as normal if it stays in the range of $0.12-0.18 \Omega$, and abnormal for any value exceeding this range, respectively.

\section{2) Multilayer Neural Network State Classifier}

The MNN is the most basic learning model among the neural network models. The MNN is a model with two or more hidden layers between the input and output layers. Complex nonlinear problems can be learned through two or more hidden layers [22].

In this study, the MNN comprises one input layer, two hidden layers, and one output layer. The rectified linear unit (ReLU) was used as an activation function for each hidden layer, and the softmax was used for the output layer. The node of the model is composed of 3600-256-256-3. The discharge voltage and state of the battery are used as the input and output, respectively. The battery status yields one of the three states: normal, warning, and fault. As a learning algorithm, the weight was updated using the RMSProp algorithm.

The RMSProp algorithm is an optimization algorithm that uses the moving average of squared gradients to normalize the gradient during neural network training. This normalization balances the step size, decreasing it for large gradients to avoid exploding and increasing it for small gradients to avoid vanishing [23]. The RMSProp algorithm is mathematically expressed as follows:

$$
\begin{gathered}
G_{t}=\gamma G+(1-\gamma)\left(\nabla_{\theta} f_{t}\left(\theta_{t}\right)\right)^{2} \\
\theta_{t}=\theta_{t-1}-\sqrt{\frac{\eta}{G_{t}-\epsilon}} \cdot \nabla_{\theta} f_{t}\left(\theta_{t-1}\right)
\end{gathered}
$$

where $\gamma$ is the decay rate (typically 0.9 ), $\theta$ is a network parameter, and $f(\theta)$ is the cost function. In this study, softmax is used. $\nabla_{\theta} f(\theta)$ is the gradient of the network, and $t$ is a time step. $\mathrm{G}_{t}$ is an accumulated squared gradient. $\eta$ is the learning rate and is set to 0.001. $\epsilon$ refers to epsilon.

The ReLU is an activation function used in neural network models [20]. This function returns 0 when it receives a negative input but returns it again for a positive $\mathrm{x}$. The ReLU solves the problem of vanishing gradients in the sigmoid and has the advantage of fast convergence. The ReLU is expressed as follows:

$$
f(x)= \begin{cases}0, & \text { for } x<0 \\ x, & \text { otherwise }\end{cases}
$$

The softmax normalizes all input values to values between 0 and 1 as output, and the sum of output values is a function that always has a value of 1 . The output comprises the number of classes to be classified. The class yielding the largest output value is used as the highest probability. The softmax function is mathematically expressed as follows:

$$
p_{i}=\frac{\exp \left(x_{i}\right)}{\sum_{k} \exp \left(x_{k}\right)}
$$

where $k$ is the number of input and output vectors and $p_{i}$ and $x_{i}$ are the $i$-th output and input values, respectively.

\section{3) Graphical User Interface (GUI) of SOH Monitoring System}

The PC GUI of the battery SOH monitoring system developed in this work is shown in Fig. 4. The GUI program was created using LabVIEW.

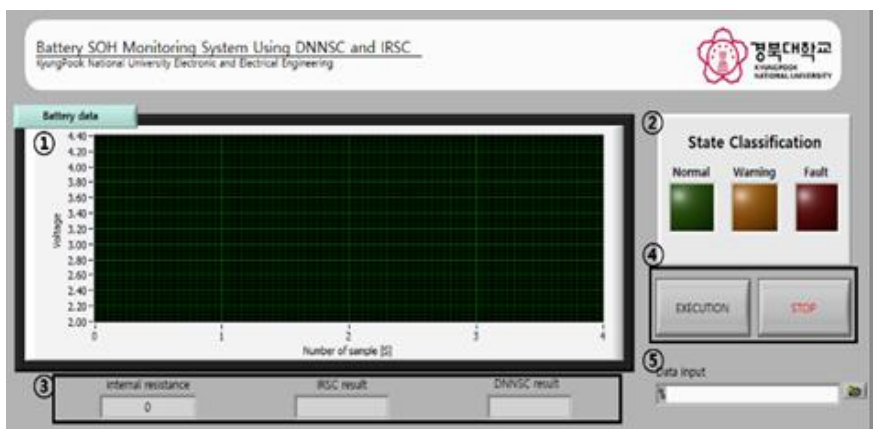

Fig. 4. GUI of the SOH monitoring system: (1) - plot of voltage from the lithium battery, (2) - output of the SOH monitoring system (normal, warning, or fault), (3) - individual results of MNNSC and IRSC, (4) - execution and stop buttons (after pressing the execution button, the MNNSC and IRSC start working and their outcomes are displayed in (3)), (5) - field to input voltage data of the battery. 


\section{EXPERIMENT AND RESULTS}

The experimental setup configuration for conducting the experiments is shown in Fig. 5. Table 2 summarizes the specifications of the battery used in the experiment.

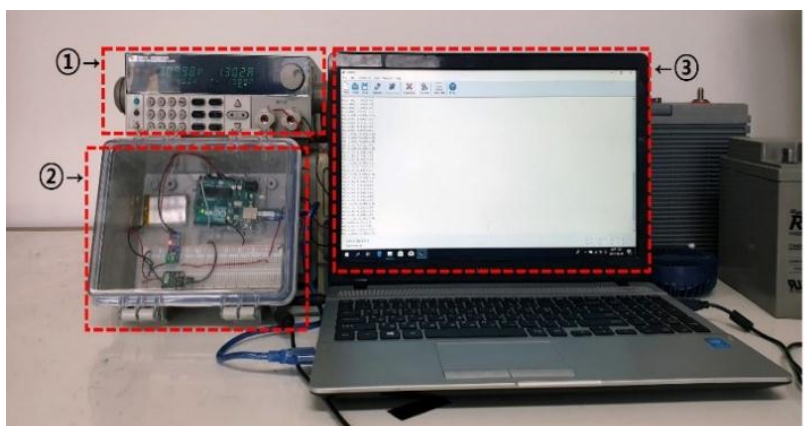

Fig. 5. Circuit configured for experiment: (1): electronic load (replaced with power supply when charging), (2): battery system (battery, current and voltage sensor, and micro controller unit (MCU) to communicate with PC0), (3): PC and serial communication with battery system to receive battery voltage and current data and monitor battery $\mathrm{SOH}$.

Table. 2. Battery internal resistance used in test

\begin{tabular}{cc}
\hline Battery type & Li-Po battery \\
Capacity & $1300 \mathrm{mAh}$ \\
Voltage range & $2.4-4.28 \mathrm{~V}$ \\
Nominal voltage & $3.7 \mathrm{~V}$ \\
\hline
\end{tabular}

To verify the performance of the proposed method, the voltage data of a lithium battery operated at a high temperature was used. The data used are shown in Fig 6. Fig 6 (a) shows the voltage data of a lithium battery used for learning. When the capacity of the battery reaches $80 \%$ of the rated capacity, it is defined as a fault. Therefore, we assumed that the state of the battery changes when the current capacity of the battery decreases by $10 \%$ from the original rated capacity. When the capacity reaches $90 \%-100 \%$ of the rated capacity, it is defined as a normal state. When the capacity reaches $80 \%-90 \%$, it is defined as a warning state. When the capacity is less than $80 \%$, it is defined as a fault state. In Fig 6 (a), patterns 1-5 were learned in a normal state. Patterns 6 and 7 were learned in a warning state. Patterns 8-10 were learned in a fault state. Fig. 6 (b) shows the discharge graphs of another battery operated in the same environment as that of the test. In Fig. 6 (b), patterns 1,2 , and 3 depict the normal state with $\mathrm{SOH}$ of $90 \%$ or more, patterns $4,5,6$, and 7 depict the warning state with $\mathrm{SOH}$ close to $80 \%$, and patterns $8,9,10$, and 11 are for the fault state with SOH less than $80 \%$, respectively.

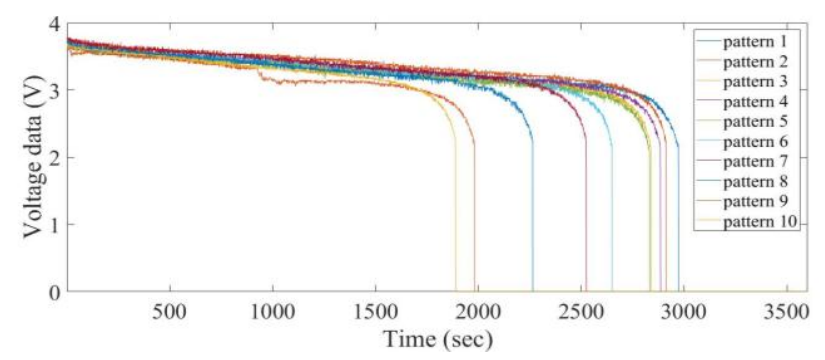

(a) The voltage data of the operated battery used in learning

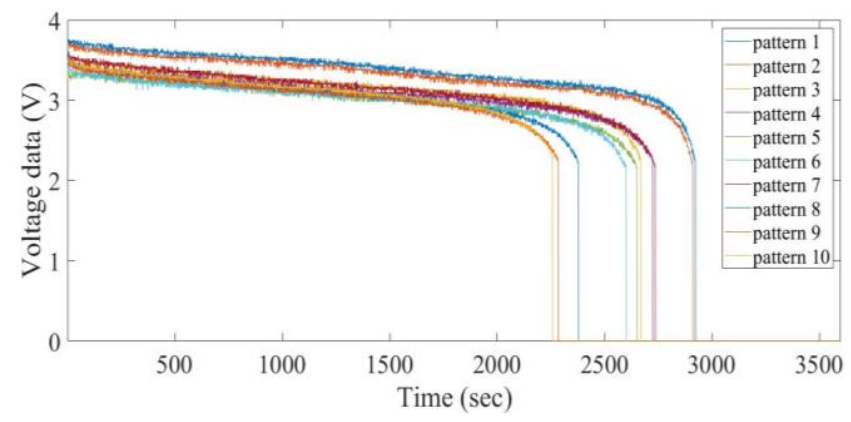

(b) The voltage data of the operated battery used in testing

Fig. 6. Lithium battery discharge graph for operation at high temperature.

Fig. 7 shows the test results by MNNSC for patterns 1 through 11 of Fig. 6 (b). The MNNSC classified patterns 1, 2, and 3 as normal, patterns 4, 5, 6, and 7 as warning, and patterns $8,9,10$, and 11 as fault states, respectively. From the results, it shows that the MNNSC method learned the characteristics of the battery well and also diagnosed battery status very well.

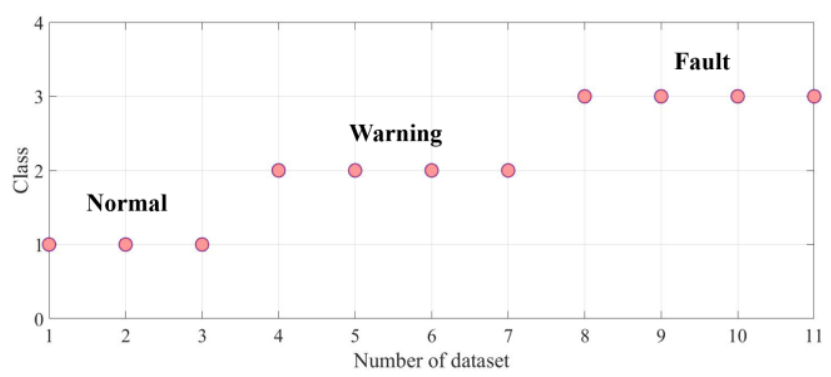

Fig. 7. SOH diagnostic test results for battery operated at high temperature.

The internal resistance of the battery used in the test in Fig. 6 (b) is shown in Table 3. The final result diagnosis from the coordinator by MNNSC and IRSC is shown in Fig. 8. It shows that MNNSC and IRSC results were diagnosed by the diagnostic rules of coordinator very well. From the results, we know that the proposed $\mathrm{SOH}$ system diagnoses the battery status was high accuracy. 
International Journal of Engineering Research and Technology. ISSN 0974-3154, Volume 13, Number 11 (2020), pp. $3240-3246$

(C) International Research Publication House. https://dx.doi.org/10.37624/IJERT/13.11.2020.3240-3246

Table. 3. Internal resistances of batteries used in the test

\begin{tabular}{ccc}
\hline Pattern & Internal resistance & Result \\
\hline 1 & 0.173 & Normal \\
2 & 0.13 & Normal \\
3 & 0.143 & Normal \\
4 & 0.208 & Abnormal \\
5 & 0.157 & Normal \\
6 & 0.257 & Abnormal \\
7 & 0.227 & Abnormal \\
8 & 0.223 & Abnormal \\
9 & 0.268 & Abnormal \\
10 & 0.252 & Abnormal \\
11 & 0.258 & Abnormal \\
\hline
\end{tabular}

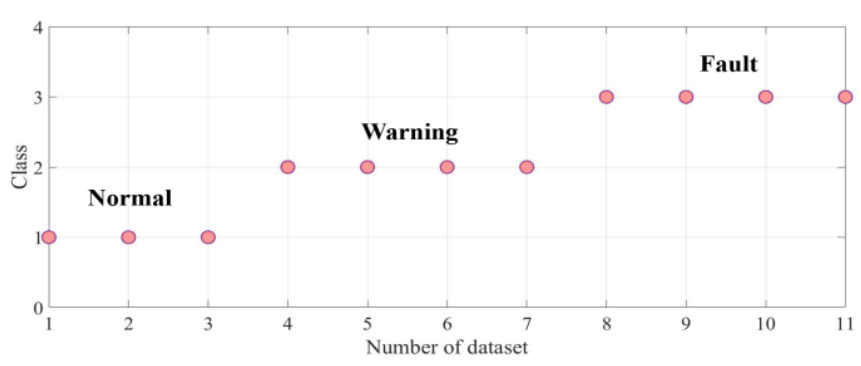

Fig. 8. Final diagnosis results from the coordinator

The performance of this system was evaluated using F1-Score [24]. The F1_Score is the harmonic mean of the precision and the recall. The precision is the ratio of correctly predicted positive observations to the total predicted positive observations, and is calculated as follows:

$$
\text { Precision }=\frac{T P}{T P+F P}
$$

where TP is the number of true positives. These are the correctly predicted positive values, i.e., when the value of the actual class is true, and the value of the predicted class is also true. FP is the number of false positives, i.e., when the value of the actual class is false, but the predicted class value is true.

The recall is the ratio of correctly predicted positive classifications to all indications of the actual class, and is calculated as follows:

$$
\text { Recall }=\frac{T P}{T P+F N}
$$

where $F N$ is the number of false negatives, i.e., when the actual class value is true, but the predicted class value is false. Variable F1_Score is defined as follows:

$$
F 1_{\text {Score }}=2 \times \frac{\text { Precision } \times \text { Recall }}{\text { Precision }+ \text { Recall }} .
$$

The accuracy is one of the most intuitive performance measures and it is simply defined as the ratio of correctly predicted observations to the total number of observations:

$$
\text { Accuracy }=\frac{T P+T N}{T P+F P+F N+T N} .
$$

$\mathrm{TN}$ is the number of true negatives, i.e., the correctly predicted negative class values, when the value of the actual class is false, and value of the predicted class is also false.

Using Equations (9)-(12), F1_Score and accuracy can be calculated using the values in Table 4, F1_Score $=2 \times \frac{1 \times 1}{1+1}=$ 1 , and Accuracy $=\frac{3+4+4}{11}=1$. The score shows that the diagnosis is perfect.

In addition, patterns 1,6 , and 9 were used to diagnose the battery status in the GUI. Patterns 1, 6, and 9 were normal, warning, and fault states, respectively, and the GUI was able to correctly classify them and produce outputs for each pattern, as shown in Figs. 9 (a)-(c).

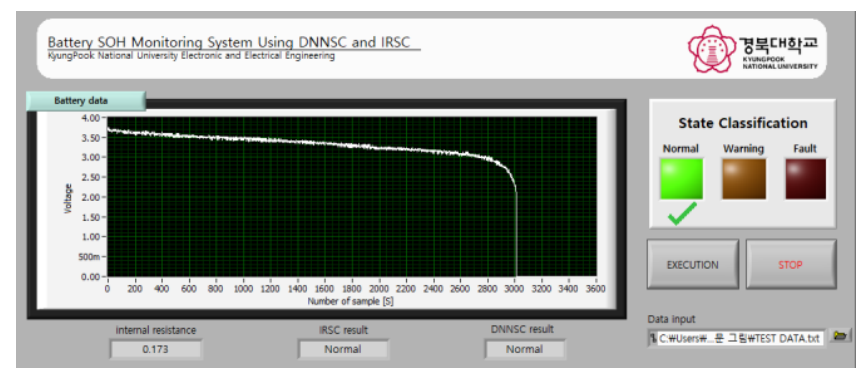

(a) Normal state

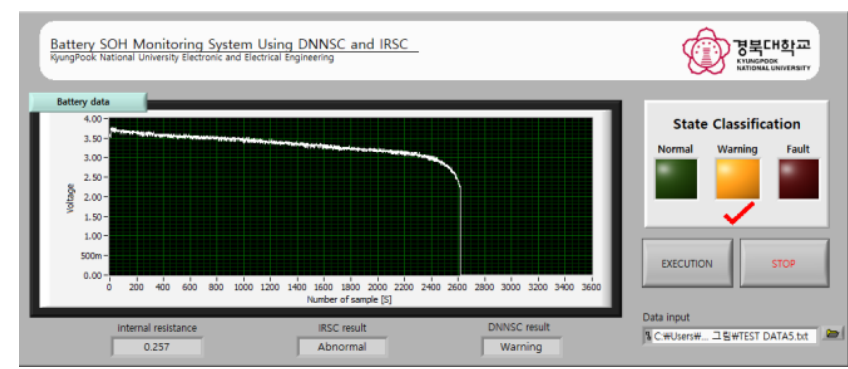

(b) Warning state

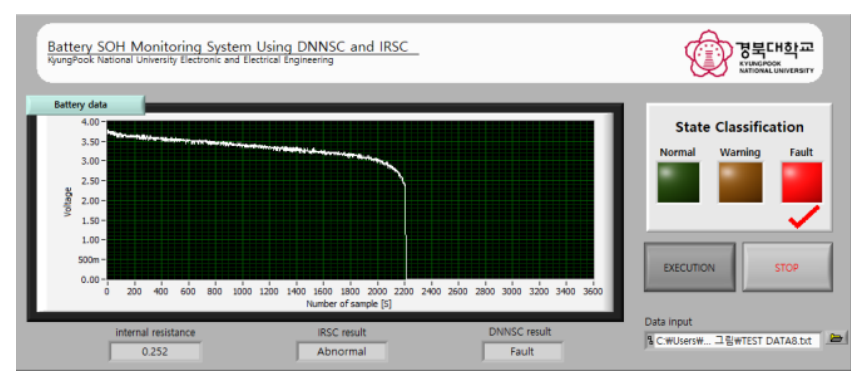

(c) Fault state

Fig. 9. SOH monitoring results for battery module. 


\section{CONCLUSION}

In this paper, the lithium battery $\mathrm{SOH}$ monitoring system based on MNNSC and IRSC was proposed. The battery was charged and discharged in a high-temperature chamber $\left(40^{\circ} \mathrm{C}\right)$, and the battery discharge voltage data obtained in the experiment were used to diagnose the fault state of the battery using the proposed method.

The experimental results demonstrated that the proposed state classifier well diagnosed the actual state of the battery by testing the discharge data of lithium battery of the same specification operated under the same experimental conditions. Moreover, a GUI for the SOH monitoring system was developed using LabVIEW that allows a user to check the status of the battery at a glance.

In the future, we will apply the proposed method based on MNNSC and IRSC for multiple battery cells because an actual lithium battery is used as a battery pack comprising multiple battery cells. Moreover, future research will significantly improve the practicality of the system.

\section{ACKNOWLEDGMENT}

This research was supported by the Basic Science Research Program through the National Research Foundation of Korea (NRF) funded by the Ministry of Education (No. 2020R1I1A3A04036615).

\section{REFERENCES}

[1] Z. Gao, C. S. Chin, J. H. K. Chiew, Jia, and C. Zhang, "Design and Implementation of a Smart Lithium-Ion Battery System with Real-Time Fault Diagnosis Capability for Electric Vehicles," Energies vol. 10, no. 10, 1503, 2017.

[2] H. R. Hwang, B. S. Kim, J. H. Kim, R. Xu and I. S. Lee, "Development of a fault diagnosis algorithm for Solar Panel," Proceedings of IEIE Summer Conference, pp. 1463-1465, 2016.

[3] T. H. Cho, H. R. Hwang, J. H. Lee and I. S. Lee, "Comparison of Intelligent Methods of SOC Estimation for Battery of Photovoltaic System, International Journal of Advanced Computer Science and Applications, vol.9, no. 9, pp. 49-56, 2018.

[4] Sidhu, Amardeep, Afshin Izadian, and Sohel Anwar, "Adaptive nonlinear model-based fault diagnosis of Liion batteries." IEEE Transactions on Industrial Electronics, vol. 62, no. 2, pp. 1002-1011, 2014.

[5] Singh, Amardeep, Afshin Izadian, and Sohel Anwar, "Fault diagnosis of Li-Ion batteries using multiplemodel adaptive estimation," IECON 2013-39th Annual Conference of the IEEE Industrial Electronics Society. IEEE, 2013.

[6] Y. Zhao, P. Liu, Z. Wang, L. Zhang and J. Hong, "Fault and defect diagnosis of battery for electric vehicles based on big data analysis methods," Applied Energy vol. 207, pp. 354-362, 2017.

[7] Z. Chen, R. Xiong, J. Tain, X. Shang and J. Lu, "Modelbased fault diagnosis approach on external short circuit of lithium-ion battery used in electric vehicles," Applied Energy vol. 184, pp. 365-374, 2016.

[8] S Park, L Zhang, and S Chakraborty, "Battery Assignment and Scheduling for Drone Delivery Businesses," 2017 IEEE/ACM International Symposium on Low Power Electronics and Design (ISLPED), IEEE, 2017.

[9] Kim, Jungsoo, et al. "Estimation of Li-ion Battery State of Health based on Multilayer Perceptron: as an EV Application," IFAC-PapersOnLine, vol. 51, no. 28, pp.392-397, 2018.

[10] Andre, Dave, et al. "Advanced mathematical methods of SOC and SOH estimation for lithium-ion batteries," Journal of Power Sources, vol. 224, pp. 20-27, 2013.

[11] Ng, Kong Soon, et al. "Enhanced coulomb counting method for estimating state-of-charge and state-ofhealth of lithium-ion batteries," Applied energy vol. 86 no. 9, pp. 1506-1511, 2009.

[12] Y. Zhang, et al. "A LSTM-RNN method for the lithuimion battery remaining useful life prediction," 2017 Prognostics and System Health Management Conference (PHM-Harbin), pp. 1-4, 2017.

[13] J. Liu, et al. "An adaptive recurrent neural network for remaining useful life prediction of lithium-ion batteries,"NATIONAL AERONAUTICS AND SPACE ADMINISTRATION MOFFETT FIELD CA AMES RESEARCH CENTER, 2010.

[14] J. Schürmann, "Pattern classification: a unified view of statistical and neural approaches,", New York: Wiley, pp. I-XVII, 1996.

[15] L. Fausett, "Fundamentals of neural networks: architectures, algorithms, and applications," PrenticeHall, Inc., 1994.

[16] Th.F. Elshatter, M.T. Elhagry, E.M. Abou-Elzahab and A.A.T. Elkousy, "Fuzzy modeling of photovoltaic panel equivalent circuit," in: Proceedings of the Photovoltaic Specialists Conference, vol. 15, no. 22, pp. 1656-1659, 2000.

[17] Wright, R. B., et al. "Power fade and capacity fade resulting from cycle-life testing of advanced technology development program lithium-ion batteries," Journal of Power Sources vol. 119, pp, 865-869, 2003.

[18] Burke, Andrew, and Marshall Miller. "Life cycle testing of lithium batteries for fast charging and second-use applications," 2013 World Electric Vehicle Symposium and Exhibition (EVS27), IEEE, 2013.

[19] Jong-Hyun Lee, Hyun-Sil Kim, In-Soo Lee "Deep Neural Network based SOH Monitoring of Battery 
International Journal of Engineering Research and Technology. ISSN 0974-3154, Volume 13, Number 11 (2020), pp. $3240-3246$

(C) International Research Publication House. https://dx.doi.org/10.37624/IJERT/13.11.2020.3240-3246

module," 2019 IEEE Eurasia Conference on IOT, Communication and Engineering (ECICE), 2019.

[20] L. Zhang, H. Peng, Z. Ning, Z. Mu, and C. Sun, "Comparative Research on RC Equivalent Circuit Models for Lithium-Ion Batteries of Electric Vehicles," Appl. Sci. vol. 7 no. 10, 1002, 2017.

[21] Hinton, Geoffrey E., Simon Osindero, and Yee-Whye The, "A fast learning algorithm for deep belief nets," Neural computation vol. 18, no. 7, pp. 1527-1554, 2006.

[22] Clevert, Djork-Arné, Thomas Unterthiner, and Sepp Hochreiter, "Fast and accurate deep network learning by exponential linear units (elus)," arXiv preprint arXiv:1511.07289, 2015.

[23] T Tieleman, G Hinton, "Lecture 6.5-rmsprop: Divide the gradient by a running average of its recent magnitude", COURSERA: Neural networks for machine learning, 2012.

[24] D. M. Powers, et al. "Evaluation: from precision, recall and F-measure to ROC, informedness", markedness and correlation, 2011. 